\section{TARGETING VASOACTIVE INTESTINAL PEPTIDE RECEPTOR SIGNALING IN PANCREATIC DUCTAL ADENOCARCINOMA FOR ENHANCED ANTI-TUMOR RESPONSE TO CHECKPOINT BLOCKADE}

${ }^{1}$ Sruthi Ravindranathan*, 'Tenzin Passang Fnu, ${ }^{1}$ Jian Ming Li, ${ }^{1}$ Rohan Dhamsania, ${ }^{1}$ Michael Ware, 'Mohammad Zaidi, 'Shuhua Wang, 'Jingru Zhu, 'Maria Cardenas, ${ }^{1}$ Yuan Liu, 'Sanjeev Gumber, ${ }^{1}$ Brian Robinson, ${ }^{2}$ Anish Majumdar, ${ }^{1}$ Shanmuganathan Chandrakasan, ${ }^{1}$ Haydn Kissick, ${ }^{2}$ Alan Frey, ${ }^{3}$ Susan Thomas, ${ }^{1}$ Bassel ElRayes, 'Gregory Lesinski, 'Edmund Waller. 'Emory University, Atlanta, GA, United States; ${ }^{2}$ Cambium oncology, Atlanta, GA, United States; ${ }^{3}$ Georgia Institute of Technology, Atlanta, GA, United States

Background Paucity of $\mathrm{T}$ cells in the immune privileged tumor microenvironment (TME) of pancreatic ductal adenocarcinoma (PDAC) is a major reason that PDAC is refractory to immune checkpoint blockade. ${ }^{1}$ In this study, we show that human PDAC tumors over-express vasoactive intestinal peptide (VIP), an immunosuppressive neuropeptide, that inhibits effector $\mathrm{T}$ cell responses and regulates chemokine receptor expression on activated T cells. $^{2} 3$ We thus hypothesized that pharmacological inhibition of VIP receptor signaling could enhance antitumor responses in PDAC.

Methods VIP levels in plasma were determined via VIP-specific enzyme immunoassay and confirmed with immunohistochemistry (IHC) of tissue sections. VIP receptor (VIP-R) signaling in C57BL/6 immunocompetent murine models of KPC, MT5 or Panc02 pancreatic cancer was inhibited by daily sub-cutaneous treatment with ANT008 or ANT308, two novel VIP-R antagonists with predicted high binding affinities to VIP receptors. ${ }^{4-7}$ Multiplex IHC or flow cytometry detected frequencies and phenotypes of intra-tumoral $\mathrm{T}$ cells across treatment groups. Results Human PDAC tumors expressed VIP by immunohistochemistry, and PDAC patients had significantly elevated plasma VIP levels when compared to healthy volunteers $(p<0.01$, figure 1). Inhibiting VIP-R signaling in combination with antiPD-1 monoclonal antibody (MoAb) synergistically enhanced Tcell dependent anti-tumor responses in murine PDAC resulting in elimination of tumors in up to $30 \%$ of the animals and increased intratumoral $\mathrm{CD} 4+$ or $\mathrm{CD} 8+\mathrm{T}$ cell density in orthotopic murine PDAC (figure 2). VIP-R antagonist+antiPD-1 combination therapy significantly increased intratumoral $\mathrm{T}$ cell activation and the proportion of tumor specific CD8+ $\mathrm{T}$ cells when compared to control $(\mathrm{p}<0.01$, figure $3-5)$. Furthermore, tumor-free mice that had been treated with VIP-R antagonist and anti-PD-1 MoAb remained tumor-free upon tumor rechallenge, indicating that combination treatment induced robust immunological memory. Interestingly, anti-PD-1 monotherapy increased expression of CXCR4 on $\mathrm{T}$ cells in tumor draining lymph nodes, a chemokine receptor that has been shown to trap $\mathrm{T}$ cells in the extracellular tumor matrix. On the other hand, combination therapy with VIP-R antagonists and anti-PD1 MoAb significantly decreased CXCR4 expression and promoted homing of adoptively-transferred GFP $+\mathrm{T}$ cells into the tumors.

Conclusions VIP-R antagonists represent a novel approach to treat PDAC. VIP and VIP-R sequences are highly conserved between humans and mice, ${ }^{8}$ and human $\mathrm{T}$ cells are activated in vitro following treatment with VIP-R antagonists. Thus, we predict comparable anti-tumor activity of the combination of VIP-R antagonist and anti-PD-1 MoAb in human PDAC patients. Further clinical development of this novel concept will require appropriate pre-clinical pharmacokinetic and toxicology studies.
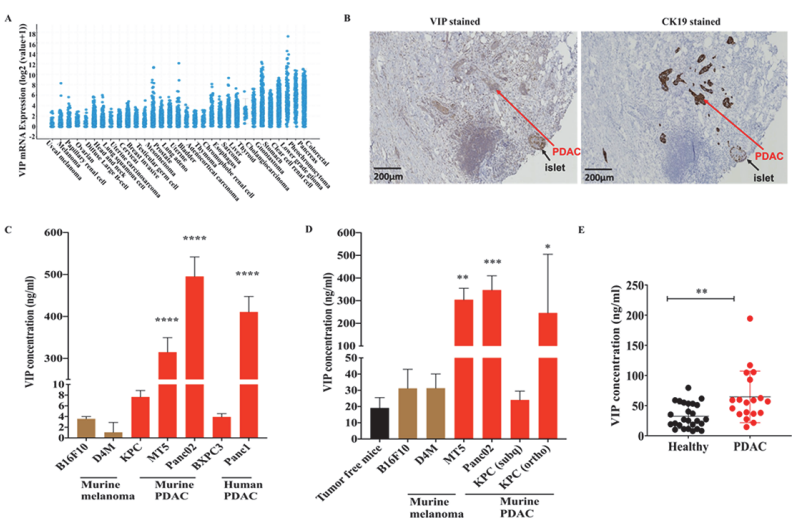

Abstract 748 Figure 1 VIP is over-expressed by PDAC. (A) VIP mRNA expression levels in various solid malignancies, as obtained from TCGA. (B) Representative images of human PDAC tumor stained with antibodies to VIP or CK19, showing VIP co-expression in islets (black arrow) and cancer epithelial cells (red arrow). Levels of VIP in (C) culture supernatants collected from murine and human PDAC cell lines cultured for 24 hours ( $n=3$ per cell line) were compared to culture supernatants from B16F10 and D4M melanoma cells; (D) plasma of mice bearing melanoma or PDAC tumors $(n=5)$ compared to plasma of non-tumor-bearing mice; (E) plasma of PDAC patients $(n=19)$ compared to that from healthy volunteers $(n=26)$. Statistical differences in $C$ and $D$ were performed by ANOVA followed by Dunnett's post-test and in $E$ were performed by student's t-test. Error bars show mean \pm SEM. ${ }^{*} p<0.05,{ }^{* *}<0.01,{ }^{* *} p<0.001$ and ${ }^{* * * *} p<0.0001$.

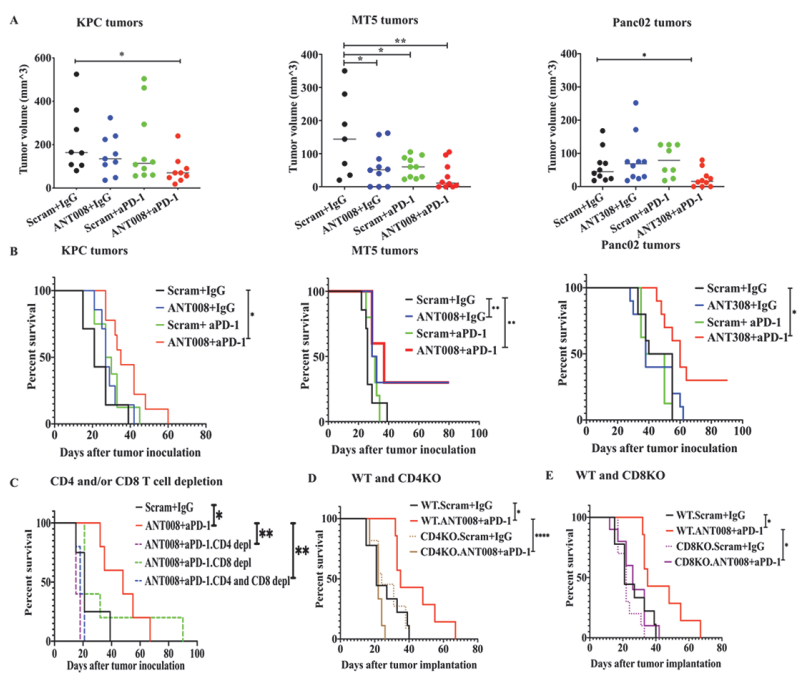

Abstract 748 Figure 2 VIP-R antagonists improve responses to antiPD-1. KPC.Luc, MT5 or Panc02 cells were subcutaneously implanted in immunocompetent C57BL/6 mice. About one week after tumor implantation, when the tumors were palpable, mice were randomized into treatment groups and treated with VIP-R antagonist and/or antiPD-1 as described in methods. (A) KPC.Luc, MT5 and Panc02 tumor volumes as measured by Vernier calipers on day 22 after subcutaneous tumor implantation. (B) Kaplan-Meier survival plots of C57BL/6 mice with subcutaneously implanted KPC.Luc, MT5 or Panc02 tumors stratified by treatment. Kaplan-Meier survival plots of (C) C57BL/6 mice receiving monoclonal $\mathrm{CD} 4$ and/or CD8 monoclonal antibodies (D) CD4KO or (E) CD8K0 mice compared to wild-type CD57BL/6 mice with subcutaneously implanted KPC.Luc tumors, stratified by treatment. Statistical differences in A were calculated by ANOVA followed by Dunnett's post-test. Solid line shows mean with in each treatment group. Statistical differences in B-E are calculated via Log-rank test. ${ }^{*} \mathrm{p}<0.05,{ }^{* *} \mathrm{p}<0.01$ and ${ }^{* * *} \mathrm{p}<0.001,{ }^{* * * *} \mathrm{p}<0.0001$. 

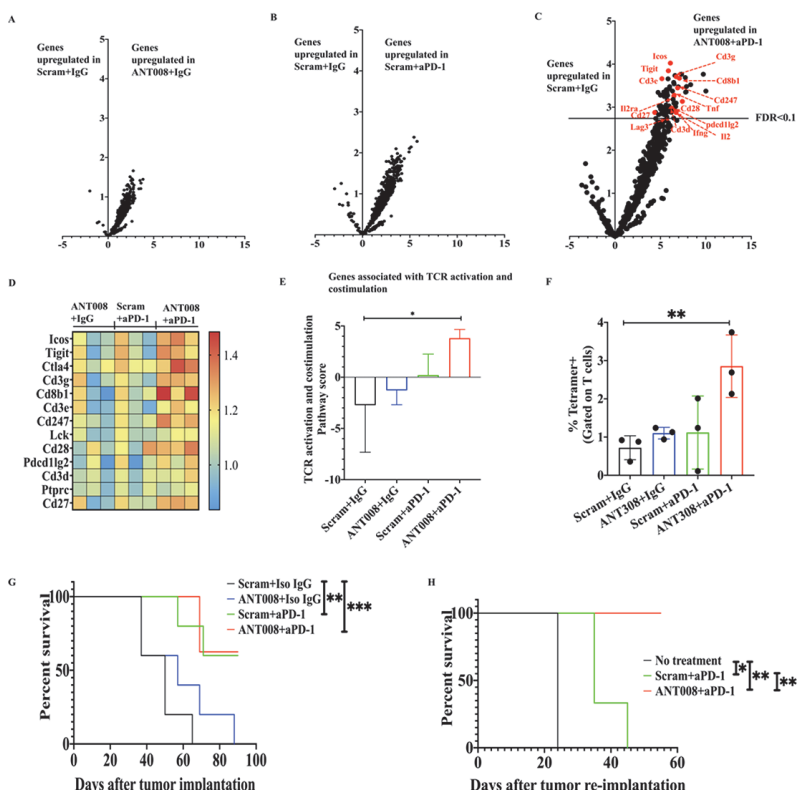

Abstract 748 Figure 3 Enhanced T cell response with combination therapy. mRNA expression in T cells isolated from subcutaneous KPC. Luc tumors in C57BL/6 mice treated with ANT008 and/or anti-PD-1 ( $n=3$ per treatment group), were analyzed via Nanostring metabolism panel. Volcano plot showing differential expression of genes in T cells from (A) ANT008+ isotype IgG (IgG) vs scrambled peptide (Scram) + isotype $\operatorname{lgG},(B)$ scrambled peptide +anti-PD-1 vs scrambled peptide + isotype IgG and (C) ANT008+anti-PD-1 vs scrambled peptide + isotype $\lg \mathrm{G}$ ( $\mathrm{n}=3$ mice per treatment group). Genes that are associated with TCR activation and co-stimulation and are at levels significantly higher when compared to Scram+ isotype IgG $(F D R<0.1)$ are labeled in red. (D) Heat map showing gene expression changes in genes associated with TCR activation and co-stimulation. (E) TCR activation and costimulation pathway score between the T cells in tumors of mice from the different treatment groups. (F) CD8+ T cells in subcutaneous KPC. Luc tumors were stained with MuLV p15E-H2Kb tetramer after 10 days of treatment with ANT308 and/or anti-PD-1 ( $n=3$ per treatment group) and analyzed via flow cytometry for percentage of tetramer+ CD8+ T cells. (G) Kaplan-Meier survival curves of subcutaneous KPC.Luc bearing mice treated with ANT008 and/or anti-PD-1 from day 3-12 after tumor implantation $(n=3$ per scrambled peptide + isotype lgG, ANT008+ isotype IgG and scrambled peptide + anti-PD-1 treatment groups; $n=8$ in ANT008 + anti-PD-1 treatment group). (H) Kaplan-Meier survival curves of tumor free mice from $G$ that were re-challenged with KPC.Luc tumors on the opposite flank ( $n=3$ per scrambled peptide + isotype lgG and scrambled peptide + anti-PD-1 treatment group; $n=5$ in ANT008 +anti-PD-1 treatment group). Statistical differences in $\mathrm{E}$ and $\mathrm{F}$ were calculated via ANOVA followed by Dunnett's post-test and in $\mathrm{G}$ and $\mathrm{H}$ were calculated using Log-rank test. Error bars show mean \pm SEM ${ }^{*} \mathrm{p}<0.05,{ }^{* *} \mathrm{p}<0.001,{ }^{* * *} \mathrm{p}<0.0001$.

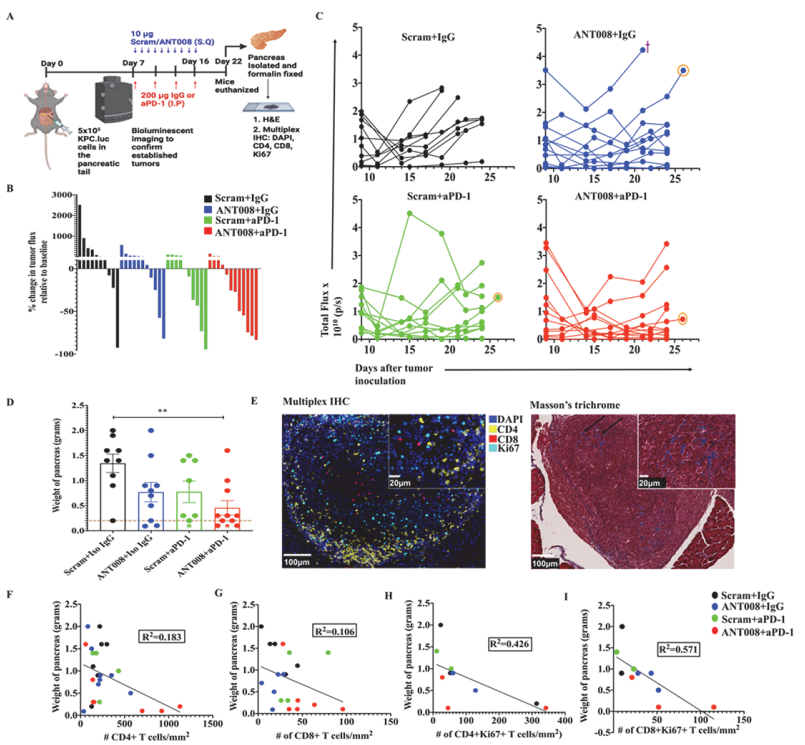

Abstract 748 Figure 4 Increased T cell density with combination therapy. KPC.Luc cells were orthotopically implanted in the tail of the pancreas of C57BL/6 mice and treated with ANT008 and/or anti-PD-1 with $n=9,10,8$ and 11 in scrambled+lgG, ANT008+lgG, scrambled tanti-PD-1 and ANT008+anti-PD-1, respectively. (A) Schematic showing orthotopic implantation of KPC.Luc cells and treatment strategy with ANT008 and/or anti-PD-1. (B) Waterfall plot showing \% change in tumor flux on day 22 relative to day 7 prior to start of treatment. (C) Total flux as measured by IVIS bioluminescent imaging in the different treatment groups. Cross symbol represents mice that were euthanized before day 25 due to ulceration of the tumor and circle symbol represent mouse that were imaged on day 26 via MRI imaging shown in supplementary figure S5. (D) Bar graph showing weight of pancreas on day 25 when the mice were euthanized. 'Star' shaped data points indicate tumor free mice and dotted horizontal line represents the average weight of healthy pancreas from naïve mice. (E) Representative multiplex IHC images (right) showing pancreatic tumors stained for DAPI (blue), CD4 (yellow), CD8 (red) and Ki67 (cyan) and trichrome staining (left) with black arrows showing blue collagen stain in the tissue. XY plot showing the correlation between number of $(F) C D 4+$ or (G) CD8+ T cells/mm2; and (H) Ki67+ CD4+ or (I) Ki67+ CD8+ T cells/ $\mathrm{mm} 2$ with weight of the pancreas with $\mathrm{n}=4$ to 6 mice per group. $P$ values in panel $D$ were calculated using student ANOVA followed by Dunnett's post hoc test (comparing each treatment group with Scram $+\lg \mathrm{G})$. Error bars show mean \pm SEM. ${ }^{*} \mathrm{p}<0.05,{ }^{* *} \mathrm{p}<0.01$. 


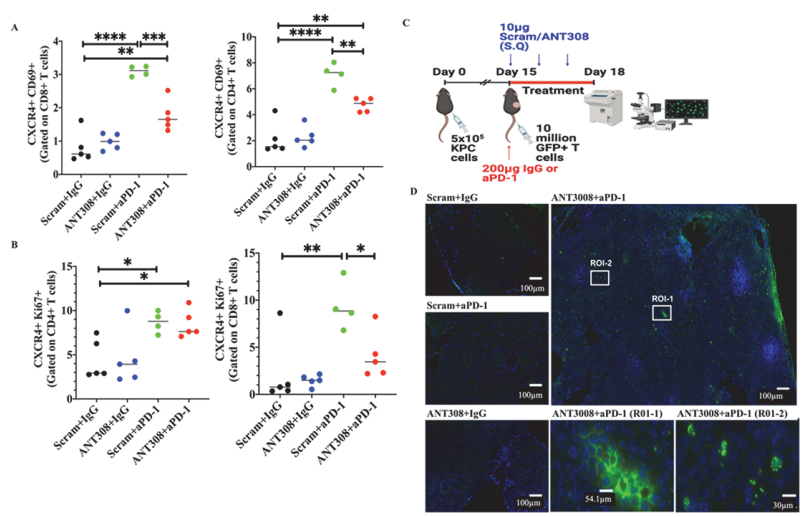

Abstract 748 Figure 5 Increased T cell homing with combination therapy. KPC.Luc tumors were subcutaneously implanted in C57BL/6 mice and treated with VIP-R antagonist and/or anti-PD-1 checkpoint therapy for 10 days after the tumors were palpable. Tumor draining lymph nodes were then analyzed for percentage of (A) CXCR4+CD69+ and (B) CXCR4+Ki67+ cells in CD4+ (left) and CD8+ (right) subsets of $T$ cells. In a separate experiment, on day 15 after subcutaneous implantation of KPC.Luc tumors, GFP+ T cells from enhanced GFP transgenic mice (C57BL/6 background) were adoptively transferred (via tail vein injections) and treated with ANT308 \pm aPD-1 for 3 days. (C) Schematic showing GFP+ T cell transfer and treatment strategy in mice with subcutaneous KPC.Luc tumors. (D) Representative Hoescht (blue for nucleus) stained tumor tissues from tumors of each treatment group. Two regions of interest (ROI) in ANT308+aPD-1 treated tumors are shown at higher magnification. Statistical differences in A and $B$ were determined via repeated measures ANOVA and Dunnett's post-test with $n=4-5$ mice per group. ${ }^{*} p<0.05,{ }^{* *} p<0.01,{ }^{* *} p<0.001, p<0.0001$.

Acknowledgements The authors thank healthy volunteers and patients for blood and/or tissue samples. The authors also thank the shared resources at Emory University, namely the Emory Integrated Genomics Core (EIGC), Emory Flow Cytometry Core (EFCC), Cancer Animal Models Shared Resource (CAMS), Cancer Tissue Pathology Core (CTP), Biostatistics Shared Resource (BSR) and Integrated Cellular Imaging Core (ICI), that provided services or instruments at subsidized cost to conduct some of the reported experiments. BioRender was used to make figure $4 \mathrm{~A}$ and 5C. This work was supported in part by Katz Foundation funding and Emory School of Medicine Dean's Imagine, Innovate and Impact (I3) venture award to Edmund $K$. Waller and NIH R01 CA207619 awarded to Susan N. Thomas. Part of the cost for the immunohistochemistry staining of tissues was covered by Winship Cancer Institute Development Discovery and Therapeutic Program Pilot funding to Sruthi Ravindranathan.

\section{REFERENCES}

1. Sahin $\mathrm{IH}$, et al. Immunotherapy in pancreatic ductal adenocarcinoma: an emerging entity? Ann Oncol 2017;28(12):2950-2961.

2. Gonzalez-Rey E, Anderson P, Delgado M. Emerging roles of vasoactive intestinal peptide: a new approach for autoimmune therapy. Ann Rheum Dis 2007;66(Suppl 3):p. iii70-6.

3. Anderson P, Gonzalez-Rey E. Vasoactive intestinal peptide induces cell cycle arrest and regulatory functions in human $\mathrm{T}$ cells at multiple levels. $\mathrm{Mol} \mathrm{Cell} \mathrm{Biol}$ 2010;30(10):2537-51

4. Li JM, et al. VIPhyb, an antagonist of vasoactive intestinal peptide receptor, enhances cellular antiviral immunity in murine cytomegalovirus infected mice. PLoS One 2013;8(5):e63381.

5. Moody TW, et al., VIP receptor antagonists and chemotherapeutic drugs inhibit the growth of breast cancer cells. Breast Cancer Res Treat 2001;68(1):55-64.

6. Moody TW, et al. A vasoactive-Intestinal-Peptide antagonist inhibits nonsmall cell lung-cancer growth. Proceedings of the National Academy of Sciences of the United States of America 1993;90(10):4345-4349.
7. Zia $\mathrm{H}$, et al. Breast cancer growth is inhibited by vasoactive intestinal peptide (VIP) hybrid, a synthetic VIP receptor antagonist. Cancer Res 1996;56(15):34869.

8. Sena $\mathrm{M}$, et al. High conservation of upstream regulatory sequences on the human and mouse vasoactive intestinal peptide (VIP) genes. DNA Seq 1994;5(1):25-9.

Ethics Approval All experimental procedures involving mice were approved by the Institutional Animal Care and Use Committee (IACUC) at Emory University. De-identified blood samples from consented patients with PDAC (IRB 00087397) or healthy volunteers (IRB 00046063) were obtained with approval from Institutional Review Boards.

http://dx.doi.org/10.1136/jitc-2021-SITC2021.748 